\title{
Unkonventionelle medikamentöse Behandlungen: Wo liegt die Grenze?
}

\author{
In zwei Entscheiden hat sich das Bundesgericht in der jüngeren Vergangenheit \\ mit der strafrechtlichen Verantwortung des Arztes für die Verschreibung von \\ Arzneimitteln befasst, welche nicht bzw. in der entsprechenden Dosierung nicht \\ zugelassenen waren (sog. Off-Label-Use). Soweit ersichtlich hat sich das Bundes- \\ gericht in diesen Entscheiden zum ersten Mal mit dem Spannungsverhältnis \\ zwischen Standardtherapie und Aussenseitermethoden auseinandergesetzt. In \\ beiden Fällen hat sich die Therapiefreiheit des Arztes durchgesetzt.
}

Korrespondenz: Dr. iur. Christoph Willi Streichenberg Rechtsanwälte Stockerstrasse 38 CH-8002 Zürich Tel. 0442082525 Fax 0442082526 streichenberg.ch

\section{Übersicht}

Beide Fälle stehen in engem Zusammenhang. Der erste Fall betraf die Behandlung mit einem in der Schweiz als Arzneimittel nicht zugelassenen Substanz (Basler Fall). Der zweite Fall betraf die Behandlung mit einem als Arzneimittel grundsätzlich zugelassenen Präparat, das jedoch in einer höheren als der zugelassenen Dosierung verabreicht wurde (Zürcher Fall, Off-Label-Use).

Trotz dieses sachlichen Zusammenhanges erfolgte die strafrechtliche Würdigung aus unterschiedlichen Perspektiven: Im Fall der Behandlung mit einem nicht zugelassenen Arzneimittel wurde dem Arzt vorgeworfen, er habe seinen Patienten die gebotene Standardbehandlung verweigert. Der Vorwurf der fahrlässigen Tötung wurde vor Bundesgericht im Gegensatz zu früheren Instanzen nicht mehr erhoben. Im Fall der Behandlung mit einer höheren als der zugelassenen Dosierung war demgegenüber zu prüfen, ob diese als fahrlässige Tötung zu qualifizieren war.

In beiden Fällen hat sich der Sachverhalt vor dem Inkrafttreten des Heilmittelgesetzes (HMG) zugetragen. Die Entscheide sind jedoch in Bezug auf die strafrechtliche Verantwortung unverändert aktuell, da das Strafgesetzbuch für diese Fragen nicht geändert hat.

Nicht zugelassenes Arzneimittel [1]

Ein Onkologe mit langjähriger Praxiserfahrung behandelte seine schwerkranken Patienten mit Lipoteichonsäure (LTA), einer als Arzneimittel nicht zugelassenen Substanz.

Am 6. September 2000 haben die zuständigen Basler Behörden dem Arzt untersagt, neue Patientinnen und Patienten mit LTA zu behandeln. In Bezug auf die bestehenden Patientinnen und Patienten wurde dem Arzt unter restriktiven Auflagen eine Bewilligung im Sinne des Compassionate Use erteilt, um die Behand-

\section{Liberté thérapeutique:} où est la limite?

Le Tribunal fédéral a récemment dû statuer dans deux affaires sur la responsabilité pénale d'un médecin ayant prescrit des médicaments non autorisés ou tout au moins pas dans la posologie en question (c'est ce que l'on appelle «un emploi non conforme»). Le Tribunal fédéral s'est donc penché pour la première fois sur le rapport de tension entre thérapie classique et méthodes parallèles, pour autant qu'il fût possible de voir clair dans ces affaires. Dans les deux cas, la liberté thérapeutique du médecin s'est imposée.

lung fortzusetzen. Vom Arzt wurde verlangt, dass er eine mit den Grundsätzen der Good Manufacturing Practice konforme Herstellung von LTA gewährleiste, sämtliche Patientinnen und Patienten vollständig aufkläre und diese ihr schriftliches Einverständnis zur Weiterbehandlung erteilen, sowie dass er die Bewilligung der Ethikkommission beider Basel zur Durchführung eines klinischen Versuches einhole. Am 4. April 2001 wurde dem Arzt die weitere Verwendung von LTA verboten, da er diese Verpflichtungen nicht erfüllt hatte. [2]

Vor Bundesgericht war nur noch der Vorwurf zu prüfen, ob der Arzt seine Patienten einer unmittelbaren, konkreten Gefährdung von Leben und Gesundheit ausgesetzt hatte, indem er ihnen die Stan- 
dardtherapie vorenthalten habe, u. a. Nolvadex ${ }^{\circledast}$ bzw. Tamoxifen.

Wegen Aussetzung ist strafbar, «wer einen Hilflosen, der unter seiner Obhut steht oder für den er zu sorgen hat, einer Gefahr für das Leben oder einer schweren unmittelbaren Gefahr für die Gesundheit aussetzt oder in einer solchen Gefahr im Stiche lässt» (aktive und passive Aussetzung, vgl. Art. 127 StGB). Weiter ist erforderlich, dass der Täter mit Wissen und Willen gehandelt hat. Die fahrlässige Aussetzung ist nicht strafbar.

Im Gegensatz zum Appellationsgericht des Kantons Basel-Stadt verneinte das Bundesgericht den Willen des Arztes, seine Patienten einer Gefährdung von Leben und Gesundheit auszusetzen. Der Arzt habe seine Patienten in der festen Überzeugung mit LTA behandelt, dass diese eine mindestens gleichwertige Alternative zu den herkömmlichen Therapien sei. In dieser Annahme sei der Arzt bestätigt worden, weil die zuständigen Basler Gesundheitsbehörden ihm örtlich und zeitlich befristete Bewilligungen für die Anwendung eines nicht zugelassenen Arzneimittels an Einzelpersonen erteilt hätten (Compassionate Use). Am fehlenden Willen zur Aussetzung vermochte der Umstand nichts zu ändern, dass der Arzt seine subjektive Überzeugung nicht auf gesicherte wissenschaftliche Erkenntnisse abstützen konnte. Der Arzt habe allenfalls «leichtfertig oder gar frivol» auf das Ausbleiben der Gefährdung vertraut, diese jedoch nicht willentlich in Kauf genommen was das Basler Appellationsgericht noch umgekehrt entschieden hatte. Eine allenfalls fahrlässige Aussetzung ist jedoch nicht strafbar, weshalb der Arzt freigesprochen wurde.

Vom Bundesgericht nicht zu prüfen war, ob der Arzt den Tod der Patienten fahrlässig verursacht hatte. Diese Frage wurde bereits vom Appellationsgericht Basel Stadt verneint. Dem Arzt konnte nicht nachgewiesen werden, dass die Standardbehandlung den Tod vermieden bzw. wesentlich hinausgeschoben hätte. Entsprechend fehlte es am Nachweis, dass die Überlebenschancen durch den rechtzeitigen Beginn der Standardtherapie hätten beeinflusst werden können.

Ebenso wenig war zu prüfen, ob sich der Arzt wegen der unbewilligten Durchführung eines klinischen Versuches strafbar gemacht hatte. Der Arzt hatte 180 Patienten einer systematischen Versuchsbehandlung zugeführt, ohne die nötige Bewilligung der Ethikkommission eingeholt zu haben. Zu jenem Zeitpunkt war das HMG noch nicht in Kraft. Für systematische Heilversuche sieht das heute geltende HMG nicht nur zwingend eine Bewilligungspflicht vor. Vielmehr kann die widerrechtliche Durchführung klinischer Versuche mit Freiheitsentzug bis zu 5 Jahren bestraft werden, auch wenn kein Patient stirbt. In Bezug auf die Durchführung klinischer Versuche kann aus diesem Freispruch nichts abgeleitet werden, wenn sich der Fall nach dessen Inkrafttreten zugetragen hätte.

\section{Nicht zugelassene Dosierung [3]}

Nach einer präoperativen Therapie mit 5-Fluorouracil (5-FU) verstarb die mit einem bösartigen Dickdarmkrebs diagnostizierte Patientin. Das Präparat war in der Schweiz als Arzneimittel zugelassen, die Anwendung erfolgte jedoch in einer höheren als der zugelassenen Dosierung.

In der Folge wurde ein Strafverfahren wegen fahrlässiger Tötung eröffnet. Das Bundesgericht hatte zu prüfen, ob der Tod der Patientin durch eine Verletzung der ärztlichen Sorgfaltspflichten verursacht wurde, da die höhere Dosierung nicht der zugelassenen Dosierung entsprach.

Das Bundesgericht stellte fest, dass das HMG die Verschreibung und Abgabe von Arzneimitteln ausserhalb der zugelassenen Indikation oder Dosierung nicht verbieten würde. Für die Beurteilung der Sorgfaltspflichten des Arztes war für das Gericht vielmehr entscheidend, dass die Aufklärungspflichten gegenüber dem Patienten erfüllt wurden. Diese sind umso höher, je weniger über die Wirkung und das Risiko eines Arzneimittels bekannt ist. Bei experimentellen Heilversuchen bestehen besonders strengere Anforderungen an die präinvasiven Aufklärungs- und Risikoabwägungspflichten.

Obwohl die höhere Dosierung nicht zugelassen war, qualifizierte das Bundesgericht diese Behandlung nicht als experimentellen Heilversuch. Angesichts einer laufenden, grossangelegten Studie über die Behandlung mit hochdosiertem 5-FU könne zwar auch nicht ohne weiteres von einem medizinischen Standard gesprochen werden. Aufgrund früherer Studien ist das Bundesgericht jedoch zum Schluss gekommen, dass diese Dosierung einer gängigen Therapieform und damit dem aktuellen Stand der Medizin entsprach. An die Aufklärungs- und Risikoabwägungspflichten waren deshalb keine besonders strengen Anforderungen zu stellen.

\section{Würdigung}

Der ärztliche Heileingriff stellt grundsätzlich eine Körperverletzung dar, die erst durch Einwilligung des Patienten gerechtfertigt wird. Die Einwilligung setzt eine hinreichende Aufklärung über alle Aspekte der in Aussicht gestellten Therapie voraus. An die ärztliche Aufklärung sind umso höhere Anforderungen zu stellen, je stärker die Behandlungsmethode von der Standardbehandlung abweicht. Hat der angemessen aufgeklärte Patient seine Einwilligung erteilt, so deckt diese nicht nur den Eingriff, sondern auch die ungewollten Folgen ab.

Ausgangspunkt für die rechtliche Würdigung ist somit das Selbstbestimmungsrecht des Patienten. Danach ist es Sache des Patienten, über die von ihm gewünschte Therapie zu bestimmen. Der Patient kann jede Heilbehandlung ablehnen, auch die allgemein anerkannte oder schulmedizinische Behandlungsmethode. Massgebend ist der Wille des Patienten und nicht das, was nach Auffassung des Arztes im Interesse 
des Patienten angezeigt ist. «Nicht alles, was medizinisch angezeigt sein könne, müsse vom betreffenden Patienten als für ihn gut und richtig betrachtet werden.»[4]

So wie der Patient jede medizinische Behandlungsmethode ablehnen kann, kann er auch einer neuen oder bisher noch wenig erprobten Therapie zustimmen. Dies gilt insbesondere dann, wenn seine Lage aus Sicht der etablierten Wissenschaft als wenig hoffnungsvoll gilt. Es gibt also keinen generellen Vorrang oder gar Exklusivität von anerkannten Behandlungsmethoden (Schulmedizin) gegenüber Aussenseitermethoden. Nichts anderes gilt für den Einsatz von Arzneimitteln gemäss Zulassung. Die Behandlung mit zugelassenen Arzneimitteln hat keinen generellen Vorrang vor nicht oder für eine bestimmte Dosierung oder Indikation nicht zugelassenen Arzneimitteln. Entscheidend ist, dass der Patient seine Zustimmung gestützt auf die angemessene Risikoaufklärung erteilt hat (informed consent).

Die ärztliche Therapiewahl ist nicht auf die zugelassenen Arzneimittel beschränkt (Therapiefreiheit). Der Arzt darf auch andere als die im Markt gängigen oder erhältlichen Arzneimittel verschreiben. Ebenso darf er zugelassene Arzneimittel anders einsetzen als dies aufgrund der Zulassung vorgesehen ist (andere Dosierung oder Indikation). Entscheidend ist, dass er die Behandlung aus medizinischen Gründen verantworten kann, und dass der angemessen aufgeklärte Patient der Behandlung zustimmt. Anzumerken ist allerdings, dass der Off-Label-Use von Arzneimitteln nur in Ausnahmefällen kassenpflichtig ist. Der Arzt muss deshalb den Patienten über die Kostenfolgen aufklären.

Am Vorrang der Therapiefreiheit haben auch das Heilmittelgesetz und die entsprechenden Ausführungsverordnungen nichts geändert. Das Heilmittelrecht sieht ausdrücklich vor, dass nicht zugelassene Arzneimittel unter bestimmten Voraussetzungen ausnahmsweise in Verkehr gebracht werden dürfen:

- Herstellung eines Arzneimittels durch eine öffentliche Apotheke in Ausführung einer ärztlichen Verschreibung für eine bestimmte Person oder Personenkreis (Arzneimittel nach Formula magistralis, vgl. Art. 9 Abs. 2 lit. a HMG). Das Arzneimittel kann ad hoc oder defekturmässig hergestellt werden. Die Herstellung durch den verschreibenden Arzt ist nicht zulässig.
- Einfuhr eines in der Schweiz nicht zugelassenen Arzneimittels mit Bewilligung der Swissmedic im Einzelfall (Art. 36 Abs. 2 AMBV). Ohne Bewilligung der Swissmedic darf der Arzt ein in der Schweiz nicht zugelassenes Arzneimittel nur in kleinen Mengen einführen, wenn in der Schweiz kein alternativ einsetzbares Arzneimittel zugelassen ist. Das Arzneimittel muss in einem Staat mit einem gleichwertigen Zulassungsverfahren zugelassen worden sein. Die ausländische Zulassung muss sich auf die gleiche Indikation beziehen.

Ebenso unbestritten ist, dass der Off-Label-Use grundsätzlich erlaubt ist, auch wenn er im HMG nicht explizit erwähnt wird.

Entscheidend ist, dass der Arzt bei der Verschreibung eines Arzneimittels die «anerkannten Regeln der medizinischen und pharmazeutischen Wissenschaft» beachtet (Art. $26 \mathrm{HMG}$ ). In Bezug auf nicht zugelassene Arzneimittel gelten deshalb dieselben Grundsätze wie sie bei anderen ärztlichen Behandlungen zu beachten sind, die mit einem Risiko verbunden sind. Welches Risiko vertretbar und damit erlaubt ist, hängt von den Umständen des Einzelfalles ab. Der therapeutische Nutzen ist dem Nebenwirkungsrisiko gegenüberzustellen. Die Schwere oder Lebensbedrohlichkeit einer Erkrankung kann ein höheres Risiko rechtfertigen. Gerade bei schwerstkranken Patienten kann es sich eher rechtfertigen, ungewöhnliche Risiken einzugehen, die nicht der «gewöhnlichen» Behandlung entsprechen. Die vorgängige Aufklärung des Patienten ist von umso grösserer Bedeutung, je weniger über die Wirkung und das Risiko eines Arzneimittels bekannt ist.

\section{Referenzen}

1 6B_40/2008 vom 20. Juni 2008, abrufbar unter www.bundesgericht.ch.

2 Beschluss des Regierungsrates des Kantons Basel-Stadt vom 8. Mai 2001 betr. schriftliche Beantwortung der Interpellation Nr. 28 vom 25. April 2001.

3 6B_646/2007 vom 24. April 2008, veröffentlicht in der amtlichen Sammlung BGE 134 IV $175 \mathrm{ff}$

4 BGE 124 IV 258, E. 2 S. 260. 